\title{
Structure of complex aluminosilicate oxide-glasses: the role of zinc intermediate element
}

\author{
A. Bernasconi ${ }^{1,2}$, M. Dapiaggi ${ }^{1}$, D.T. Bowron ${ }^{3}$ G. Agostini ${ }^{2,4}$ and A. Pavese ${ }^{5}$ \\ ${ }^{1}$ University of Milano, Milano (Italy), ${ }^{2}$ ESRF The European Synchrotron, Grenoble (France), ${ }^{3}$ ISIS Pulsed Neutron and Muon Source, \\ Didcot (UK), ${ }^{4}$ ALBA Synchrotron, Barcelona (Spain), ${ }^{5}$ University of Torino, Torino (Italy),
}

andrea.bernasconi@hotmail.it

Aluminosilicate-based oxide-glasses are natural materials forming volcanic magmas [1] and frequently the main constituent of manufactured products like ceramic glazes, fiber optic materials and, more recently, biocompounds [2]. To characterise the atomic structure of these materials requires techniques sensitive to the very local structural environment, like spectroscopies (i.e. Nuclear Magnetic Resonance - NMR, Extended X-ray Absorption Fine Structure - EXAFS) and scattering methods (i.e. Total Scattering), due to their lack in periodic order that prevents the application of conventional crystallography. The oxide-glass structure is shaped by silicon centered corner-sharing tetrahedra, which can be combined, depending on composition, with aluminium centered motifs, while large cations like sodium, potassium and calcium tend to depolymerize the network, affecting, in this way, some of the glass properties, such as thermal expansion and glass transition temperature, as demonstrated in a previous study [3]. The glass structural complexity increases when their composition involves some intermediate element, like zinc and beryllium, whose role in the network can vary as a function of the bulk composition, see [4] for some examples. This is the case of the present study that is based on a structural modeling of 2 series of different aluminosilicate-based oxide-glasses with different zinc amounts ( 3 samples each series). These samples have been prepared by melt-quenching route at $1350^{\circ} \mathrm{C}$ and then measured by combining EXAFS spectroscopy (BM 23 beamline, ESRF, France) with both neutron (SANDALS instrument, ISIS, UK) and synchrotron (ID11 beamline, ESRF, France) Total Scattering data. Zn K-edge EXAFS has been applied at the beginning, in order to evaluate some of the bond distances and the $\mathrm{Zn}$ geometrical environment, and this information is used later as constraints for total scattering data modelling, performed by the Empirical Potential Structure Refinement (EPSR) method [5]. The refinements show good residuals, as displayed in Figure 1 (on the left-hand side) and the results indicate that zinc is mostly 4-fold coordinated, but with some 3-fold, 5-fold and 6-fold species. In such complex glasses, therefore, the parameters describing the polymerization degree, like NBO (Non-Bonding-Oxygens), BO (Bonding Oxygens) and triclusters are not predictable by theoretical models, based on prior assumptions of the structural role of zinc. Figure 1 (on the right-hand side) shows the variations of $\mathrm{NBO}$ with $\mathrm{ZnO}$ mole fraction, comparing the results of this work and of theoretical calculations. Furthermore, the data modelling gave access to a wide number of structural parameters like bond angles, cluster size and cation charge compensating characteristics, that are valuable for further structure-properties studies.
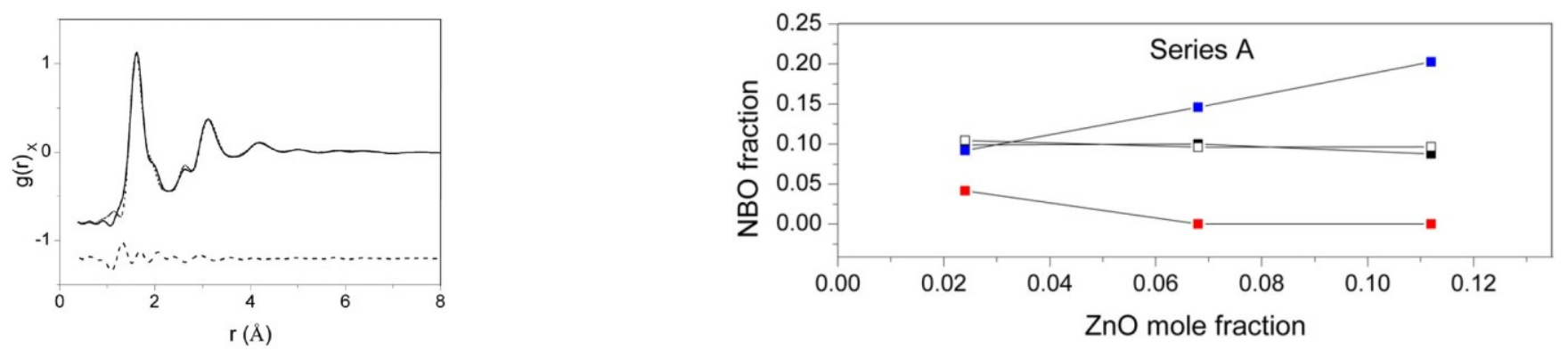

Figure 1. On the left, example of EPSR fit (for x-ray data) of one glass samples. On the right, non-bridging oxygen (NBO) polymerization parameter obtained by EPSR (black and white squares represent neutron and x-ray results, respectively) and by theoretical calculation (blue and red square are assuming zinc as network modifier and network former, respectively).

[1] Petrini, R., Forte, C., Orsi, G. , Piochi, M., Pinzino, C. \& Pedrazzi, G. (2001). Contrib. Mineral.Petrol. 140, $532-542$.

[2] Yoo, S., Basu, C., Boyland, A:, Sones, C., Nillson, J., Sahu, J. \& Payne, D. (2007). Opt. Lett. 32, 1626-1628.

[3] Bernasconi, A., Dapiaggi, M., Bowron, D.:, Ceola, S. \& Maurina, S. (2016). J. Mater. Sci.. 51, 8845-8860.

[4] Shelby, J.E. (2005). Introduction to Glass Science and Technology. Cambridge: RSC.

[5] Soper, A.K. (2010). ESPR shell: a user guide. Didcot: ISIS Disordered Material Group.

Keywords: oxide-glass structure; total scattering; Pair Distribution Function; EXAFS; 